\title{
Machines as Teammates: A Collaboration Research Agenda
}

\author{
Isabella Seeber ${ }^{1}$, Eva Bittner ${ }^{2}$, Robert O. Briggs ${ }^{3}$, Gert-Jan de Vreede ${ }^{4}$, Triparna de Vreede ${ }^{4}$, Douglas Druckenmiller ${ }^{5}$, Ronald \\ Maier $^{1}$, Alexander B. Merz ${ }^{1}$, Sarah Oeste-Reiß ${ }^{6}$, Nils Randrup ${ }^{7}$, Gerhard Schwabe $^{8}$, Matthias Söllner ${ }^{6,9}$ \\ ${ }^{1}$ University of Innsbruck \\ firstName.lastName@uibk.ac.at \\ ${ }^{2}$ University of Hamburg \\ bittner@informatik.uni- \\ hamburg.de \\ ${ }^{3}$ San Diego State University \\ rbriggs@mail.sdsu.edu \\ ${ }^{4}$ University of South Florida
gdevreede@usf.edu,
tdevreede@usf.edu \\ ${ }^{5}$ Western Illinois University \\ da-druckenmiller@wiu.edu \\ ${ }^{6}$ University of Kassel \\ oeste-reiss@uni-kassel.de \\ soellner@uni-kassel.de \\ ${ }^{7}$ AVT Business School \\ nils@randrup.info \\ ${ }^{8}$ University of Zurich \\ schwabe@ifi.uzh.ch \\ ${ }^{9}$ University of St. Gallen \\ matthias.soellner@unisg.ch
}

\begin{abstract}
Humans will soon need to adapt to a collaborative setting in which technology becomes a smart collaboration partner that works with a group to achieve its goals. It is therefore time for collaboration researchers to explore the vast opportunities afforded by smart technology and to test its utility for enhancing team processes and outcomes. In this paper, we take a long view on the implications of smart technology for collaboration process design, and propose a research agenda for the next decade of collaboration research. We create a reference model to frame the research agenda.
\end{abstract}

\section{Introduction}

Where current collaboration technologies support our team efforts, artificial intelligence technology (AI) combined with other smart technologies may soon join our efforts as a teammate. We already use Gmail, Skype, Google Docs, Dropbox, Thinktank, and the like, to communicate, to reason together, and to share information. Collaboration engineers use these tools to create technology-supported collaborative work practices that non-experts with little or no training can follow to gain discontinuous improvements in teamwork. What could happen, though, if smart technology could be your teammate? What should happen?

As AI matures, these questions move from esoteric curiosity to pragmatic opportunity. The impact of AI on business and society is already discernable. IBM's Dr. Watson ${ }^{1}$ already helps oncologists to analyze symptoms with state-of-the-art knowledge, run a

\footnotetext{
1 See https://www.ibm.com/watson/health/oncology-andgenomics/oncology/
}

patient's history, and make a diagnosis. Internet of Things (IoT) provides many new sources of information, and new devices with which to collaborate with each other. In the near future, the nature of organizations and the nature of collaboration may, as a consequence, change. Will teams of autonomous, intelligent digital agents complete collaborative tasks that were earlier either exclusively performed by humans, or perhaps that could not have been done in the past? Will sensor networks capturing real-time data enhance and be represented in our virtual collaboration environments?

Our understanding of collaboration in the form of theories, methods, and technologies has advanced tremendously over the past two decades. But there are also clear limitations to our body of knowledge, given the new technologies. We need a better understanding of the new ways collaboration may work when smart technologies join the team. We define smart technologies as those that draw inferences from information, augment available information by discovering new, relevant information, and find new insights in existing information, and participate in the cognitive decision-making process with human actors. These technologies provide an unprecedented opportunity for the international collaboration science community to discover new phenomena and new effects, to develop broader and deeper theoretical understandings of collaboration, to invent new approaches, to establish best practices, and investigate the ways the technology affects teams, organizations, and society.

We should take a long view of collaboration to understand what needs to be done today to prepare for a future where the design of human-machine 
teamwork may be central to the success of the organizations whose nature may be changing in response to smart technologies [1]. In order to do so, we need a research agenda that gives special consideration to the design of collaboration using these new technical capabilities. In this paper, therefore, we address the question: What research should collaboration researchers pursue to prepare for machines as teammates?

This paper contributes a reference model that helps structure our understanding of the collaboration opportunities and pitfall pertaining to the emergence of smart technologies, and to guide future collaboration research and design.

\section{Methodology}

Fourteen researchers from North America, Europe, and Asia joined forces to tackle this challenge. All have made substantial collaborations to the collaboration literature with seniors contributing to the domain for more than a decade. We used a 4-step procedure to address our question (see Figure 1). We began the initiative with a one-day face-to-face workshop at HICSS 2017, then continued the inquiry with asynchronous coordinated work punctuated by bi-weekly plenary web-conferences.

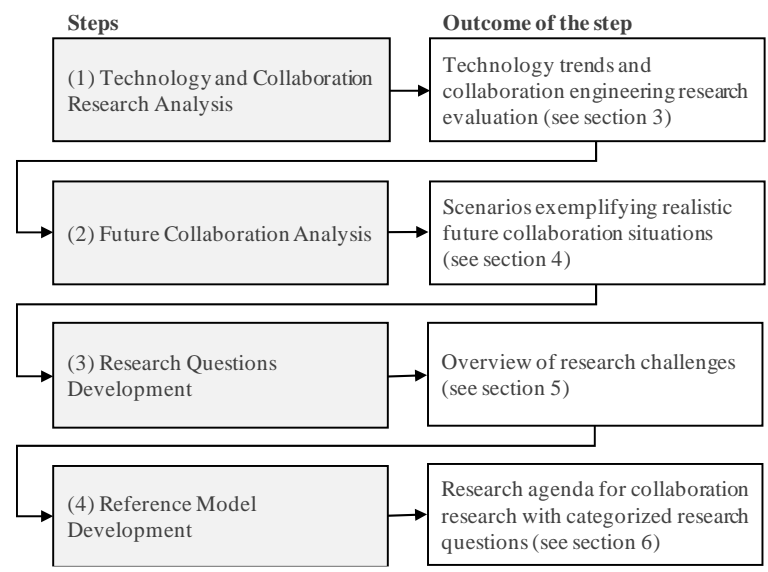

Figure 1. Research methodology overview

Technology and Collaboration Research Analysis. First, we reviewed the literature to develop a list of technologies with the potential to disrupt the way humans currently work and collaborate. We reviewed the latest collaboration technology and collaboration engineering developments, and considered their potential impact on collaboration in contexts ranging from small groups to crowdsourced collaboration among tens or hundreds of people (see section 3).
Future Collaboration Analysis. With the analytical results as a foundation, we employed a Usability Engineering approach called scenario-based design [2], [3] to identify and develop future collaboration scenarios. This phase consisted of creative efforts by a sub-group of researchers to identify and describe how smart technology creates new opportunities in the form of likely future collaboration settings. The other half of the research group provided critical feedback on the scenarios, which were subsequently altered and improved. Each scenario describes a context, specifies the agents (actors), and describes the goal, observable actions, dynamics, and events [2]. The results of the analysis is a carefully selected set of scenarios (see section 4) describing fictive but realistic future collaboration situations.

Research Questions Development. Third, we generated a number of research questions around each of the scenarios. From these we synthesized several research challenges. Based on a review of smart technology and collaboration engineering research as well as an analysis of the scenarios, we engaged in an iterative process of identifying relevant research questions which would advance scientific-based collaborative insights. In bi-weekly virtual meetings using collaborative technologies, we conducted individual reflection and small group discussions identify a larger, broader set of research questions reflecting the scope of research needed. The outcome was a set of diverse and uncategorized research questions and a set of related research challenges to help focus the attention of collaboration researches from many disciplines on the coming challenges (see section 5).

Reference Model Development. Fourth, we adopted an inductive approach and consolidated the research questions by organizing, converging, and synthesizing the research questions into related themes. Based on the emerging themes, we then identified and visualized a conceptual model that could represent associations between the themes and exemplary research questions for better comprehension. Based on the reference model, we then engaged in a last iteration of research question identification and generated new, generalized, detailed questions or adapted old, and deleted redundant research questions. The outcome of the reference model development was a list of research questions organized by themes (see section 6).

\section{Technology Trends and Collaboration Engineering Analysis}

In this section, we review current technology 
trends especially "smart technologies" and collaboration engineering's impact on collaboration practice and research.

\subsection{Technology Trend's Impact on Collaboration}

The rise of computer-based GSS in the 1980s fostered discontinuous improvements in team performance under some conditions [4]-[6]. GSS tools could be used to restrict people to productive actions they wanted to take and restrict them from unproductive actions they did not want to take. That reduced cognitive load and enhanced joint reasoning. GSS allowed for anonymous contributions when it was useful, e.g. during ideation, and during idea evaluation. GSS supported co-creation and refinement of complex bodies of knowledge, and supported building consensus around proposed courses of action [7], [8]. Drawing on a prominent report on emerging technology [9], we identified five technologies may soon have major impact on businesses. We analyzed these produce to the following assessments of their potential:

Artificial intelligence (AI). AI describes the capability of a machine to imitate intelligent human behavior [10]. AI has a long list of potential impact points on collaboration and we consider this technology as the one that will impact collaboration design and execution the most.

Augmented reality (AR)/Virtual reality (VR). AR adds information to the physical world through audio, visual, and/or sensory elements. AR is different from VR as VR reproduces reality within an immersive environment. AR may influence collaboration especially through its communication and presentation capabilities. VR may impact collaboration in the form of virtual meeting and interaction spaces, which can potentially substitute both physical and current computer/mobile device based interaction platforms.

Internet of Things (IoT). Smart artifacts and objects with sensors, computer technology, and software, which collect and exchange data over the Internet. IoT has some potential to influence collaboration especially through its gathering and sharing abilities of information, e.g. physical voting cube. When connected to artificial intelligence, this could enhance the value of IoT for collaborative efforts in (creative) industry and other economic sectors.

Robots. Physical, electro-mechanical machines automate, augment, or assist human activities

\footnotetext{
${ }^{2}$ We refer to the collection of intelligent cognitive assistants augmenting our collaboration as smart technology.
}

autonomously or by instructions. Robots may impact collaboration, especially through their abilities to perform physical collaborative tasks and appear as physical collaboration agents.

3D printing. Manufacturing techniques used to create three-dimensional objects based on digital models printing successive layers of materials on top of each other like plastic, metal, glass, organic materials or a combination of these. $3 \mathrm{D}$ printing is likely to have some but limited impact on collaboration, especially through its ability to immediately create or re-create objects during collaborative innovation or repair processes.

These technologies all have some potential to impact collaboration. However, we deem "smart" technologies like AI alone or in combination with other of the about technologies (Robots, IoT, VR) as the one technology trend that most likely to affect collaboration in the years to come. It therefore warrants a closer analysis.

\section{2 "Smart" Technology's ${ }^{2}$ Impact on Collaboration}

Some industry leaders and researchers argue that within five years all major business will rely on socalled cognitive technologies [11], which are in essence AI enabled "smart" technology. Human intelligence will be augmented with cognitive technologies such as natural language processing, neural networks, and deep learning ${ }^{3}$ which will substantially change how humans work together, make decisions, and manage organizations. In many cases, humans will hand off tasks to machines and back again [1] and machines will know more than humans do [12]. Smart technologies like AI will help to collect, understand [13], judge, reason, explain the implications of options, and in some cases, make choices [14] for knowledge-intensive tasks. They will mimic some aspects of human learning [15]. We will have teammates that are not human but smart robots; giving us advice or even giving us instructions [12]. In contrast to humans, machines have unlimited attention spans, millisecond reflexes, and infallible memories [16]. Smart technology such as AI is good at generating new combinations of preexisting elements [17] or performing classification activities based on pattern recognition [15]. Humans prevail particularly in creative ideation [17]. In these cognitive activities, humans are able to come up with unique and original ideas [18] because of their abilities in general pattern recognition (not specialized towards a specific

\footnotetext{
${ }^{3}$ We consciously abstain from providing a more details on these technologies, because we focus on their purpose in supporting collaboration rather than specific approaches.
} 
domain) and their complex communication abilities [17] to understand and solve a problem. Nonetheless, AI in connection with other technologies such as robots, and AR/VR will most likely become vital partners supporting us in knowledge-intensive collaboration tasks.

\subsection{Collaboration Engineering's Impact on Collaboration}

In the early 2000s, researchers of collaboration and GSS noticed that they were using similar techniques and tools over and over again to engage teams in successful collaboration. The design of such ITenabled techniques and the study of their effects resulted in a discipline which today is known as Collaboration Engineering (CE) [19]. The goal of CE is to provide guidance for designing effective collaborative work practices for high-value recurring tasks [20]. To achieve this goal, CE researchers have developed an impressive number of models, methods, and tools to guide the systematic design of effective IT-supported human collaboration processes [20]. One example is represented by the six patterns of collaboration in which a complex collaboration process is divided into smaller activities with activity goals [19]. For each of the six patterns of collaboration, CE research has developed a collection of techniques, also known as ThinkLets, that help facilitate reaching the activity goals using collaboration technology [21]. These codified techniques aim at enabling non-collaboration experts to execute the standardized and highly effective collaboration process designs without ongoing support from or under direct leadership by professional facilitators [22]. Other developments include for example the Six Layer Model of collaboration [23] or the five ways of thinking framework.

\section{Collaboration Scenarios}

Based on the smart technology and CE analysis, we developed several scenarios and selected four to illustrate different collaborative situations with future smart technology. We have selected these since they represent various core collaboration events which leverage one or more smart technologies. The scenarios' purpose is to exemplify future collaborative situations which then serve as the basis to deduce research challenges and develop a set of critical research questions for the future.

Scenario 1: Cognitive Computing in Workshops The board meeting had been scheduled for $1 \mathrm{pm}$. The main agenda item is the monthly risk review. The chief marketing officer Sandra presents slides on recent image studies performed by a marketing research company. After the presentation, the cognitive system Watson smiles. He has been invited to challenge the insights presented by the human presenter with questions and new information. And it is easy to challenge Sandra's presentation: "What about the driving restrictions an increasing number of German cities will impose on diesel cars? Will our positive image as a future electric car company really balance the fact that we are currently selling 45 percent of our cars with a Diesel engine." Sandra begins to sweat - she had not paid attention to this very recent issue. She whispers at the cognitive assistant Butler: "Please, find out quickly how many of our customers would be subject to diesel restrictions in German cities and how that compares to other carmakers." Butler's immediate answer is sufficient to put this issue on the risk watch list but no immediate actions are taken.

\section{Scenario 2: Crowd Testing}

Luke sets up a new project for bug testing on the crowd-testing platform. Quickly, the first bug reports come in and are collected by an AI that forwards genuine and probably suitable reports to Luke. The AI constantly observes Luke's way of processing bug reports. Since its inception it has extended its original database of debugging algorithms and can solve programming errors in the bug reports independently and automatically. This time, most of the bug reports can be solved by the AI on the test system. The code changes are automatically put in the cue for the next update on the life system so that future crowd testers will no longer see the bugs. The AI forwards 5 genuine and suitable reports to Luke who then processes the first reports. He takes a long time to read it in detail. Luke likes that he does not have to deal with all the redundant bug reports that used to come in because testers sent reports over and over again or multiple testers worked on the same bug. After finishing up with the other bug reports, Luke checks the filtered bug reports because he knows that also his AI could make mistakes. Luke tells the AI to show all bug reports that have been filtered based on a probability score of below 0.95. Twenty reports show up and within 10 minutes he identifies two new important bug reports that had been wrongly categorized as redundant even though they had not been solved in the system. He is quite glad to have also checked the filtered list.

Scenario 3: Additive Manufacturing The farmer Jim and his neighbors encountered a 
serious problem with their tractor's performance in fall operations. They all go into the lab and activate JohnDAI, whose avatar looks remarkably like the original 1837 blacksmith and inventor. "We got a problem," says Jim. "Part number 456-78A in the engine assembly keeps failing." JohnDAI responds with, "Hold on a sec ; I'll call up the specs and performance records from you and your neighbor's cognitive assistants JimAI, OleAI, and SvenAI." JohnDAI frowns, "Yep, I see the problem, but it's intermittent and as usual in a complex system I can't determine the actual root problem. What do you think?" Ole says, "It's the damn plastic parts," Jim says, "It's the fuel!" Sven yells, "It ain't the fuel, it's the new software upgrade!" JohnDAI notifies the engineering team responsible for the fuel system of the problem, and they join the virtual conferencing system. Tina, the lead engineer from the German engineering innovation center in Mannheim, says, "Hi guys, what's the problem?" JohnDAI briefs the engineering team on the symptoms and displays the technical schematics, materials analysis, and specifications. The team considers the materials problems and fuel analysis data direct from Farmer's Coop. As a group they use the sophisticated CAD software to collaboratively modify and test the current part design in an attempt to digitally produce the problem. In an analysis of the fuel mix, Tina's materials engineer, Paul, notices that the ethanol content has a potential reaction with the plastic polymer of the part. This factor was not taken into consideration in the original design. They make adjustments to the materials specifications and print the new parts that same day for field trial.

Scenario 4: Crowd workers in fluid collaboration environments

John is the Creative Director of his advertising agency AdvertNice and discusses campaign aims with customer Amanda from TelCo. On behalf of Johns instruction, the AI Glyder combs social media and online data sources to discover the emotions, values, and utility current customers ascribe to TelCo's services. Glyder also identifies TelCo's key competitors and performs similar analyses on their customers. Then, he identifies non-customers who share the same emotions, values, and utility in order to propose new market segments. The result is a campaign profile that John uses to set up a project for generating tag lines around the emotions, values, utility, and customer segments. John's other AI for project and teamwork management called Fluid breaks down the campaign project into individual work packages and deliverables. Based on the skill required, it automatically hires available crowd workers with matching skills from a number of different crowdworking platforms. After Fluid has acquired 50 crowd workers from around the world and automatically organized them into smaller teams of 4 to 6 people per work package, John is now ready to kick-off the campaign project in Fluid. Depending on the work package's tasks, Fluid creates a distinct collaboration environment by orchestrating from the communication channels those that fit to the task most ideally and makes suggestions about how to start collaborating. As the crowd workers move along, Fluid adapts the collaboration environment and adds different communication channels to the team, infers minutes from chat protocols, and suggests to-dos to team members.

\section{Research Challenges}

The analysis of smart technologies and CE's impact on collaboration, as well as future scenarios of collaborative situations, opened opportunities for a variety of important research questions.

In future collaboration, smart technology may lead to novel modes of (crowd) work, allowing highly flexible scaling of businesses, but will potentially also threaten existing business models and disrupt traditional work settings. It would be useful to investigate the degree to which humans and technologies understand their roles (Scenario (Sc.) 4) and examine the allocations of control (Sc. 2) between humans and machines. The new technologies afford substantial increases in the amount of relevant information. This calls for research on how information can be discovered, exchanged and understood among multiple (inter)connected smart technologies (Scs. 3 and 2) and humans. Smart technology needs to be embedded in communication infrastructure (Sc. 4) that lets smart technology agents to provide task-related suggestions and decision guidance based on collected, analyzed, and synthesized data on human interactions. Teams may be able to add diversity to their collaboration if they let smart technology mimic team behaviors (Sc. 1), such as the devil's advocate. Numerous sociotechnical operational question marks remain about how the technology would have to be designed and used to make it both effective and acceptable. To augment our cognitive processing, smart technology should provide accurate information (Scs. 1, 2 and 3), but, given the volume of anticipated information, may also need to learn independent of human feedback (Sc. 8) while collaborating with humans. Information provided by smart technology may become an integral part of the human decision-making process. There are a number of questions to be answered about how such 
information can be validated, and the conditions under which humans should trust or mistrust it (Scs. 1 and 2). As technology agents gain cognitive capabilities and so task autonomy, it will be useful to discover the domains where humans excel, the domains where AI excels, and the domains where the combination excels either alone (Sc. 3). It is not yet clear the degree to which the new technologies will make opportunities for human creativity more sparse or more numerous.

It may be possible to design collaboration processes between humans and smart technology such that humans feel empowered or disempowered by the association (Sc. 3). We do not yet know the degree to which people might perceive those changes as positive or negative, or the consequent design principles for addressing the politics of non-human teammates. The use of independent smart technology for some tasks may raise questions of auditability and rationale (Sc. 2). There are unanswered ethical and pragmatic questions about who should be held accountable for unfavorable outcomes. There is also the risk that false information provided by smart technology might be trusted blindly (Scs. 1 and 2). Therefore, questions of credibility assessments of smart technology become important (Sc. 2).

\section{Research Agenda for Collaboration}

The questions raised in this section are but a brief sample of the hundreds of diverse questions that emerged from our discussion. We therefore structured the emerging issues into a reference model (see Figure 2) to create a thematic overview of the research questions we developed. The reference model is divided into three meta-themes. Each is elaborated with several sub-themes, and seeded with selected research questions.

\subsection{Collaborative Sphere (Meta-Theme A)}

The first theme that emerged from the analysis of the research questions was that some research questions (see Themes 1-4 below) were similar with respect to the actions, execution, and behavior in collaboration situations and not the design of the collaboration process. We refer to this mega-theme as the Collaborative Sphere.

Collaborative Boundaries (Theme 1) describes the research questions related to the overarching environment and framework conditions under which the collaboration execution constructs interact with each other. The boundaries cover all environmental or situation specific factors that cannot be manipulated within the time-frame of the collaboration planning and execution. Therefore, boundaries can be considered as rigid and uncontrollable restrictions of the collaborative events. However, the boundaries can act as restrictions or opportunities depending on how they are utilized.

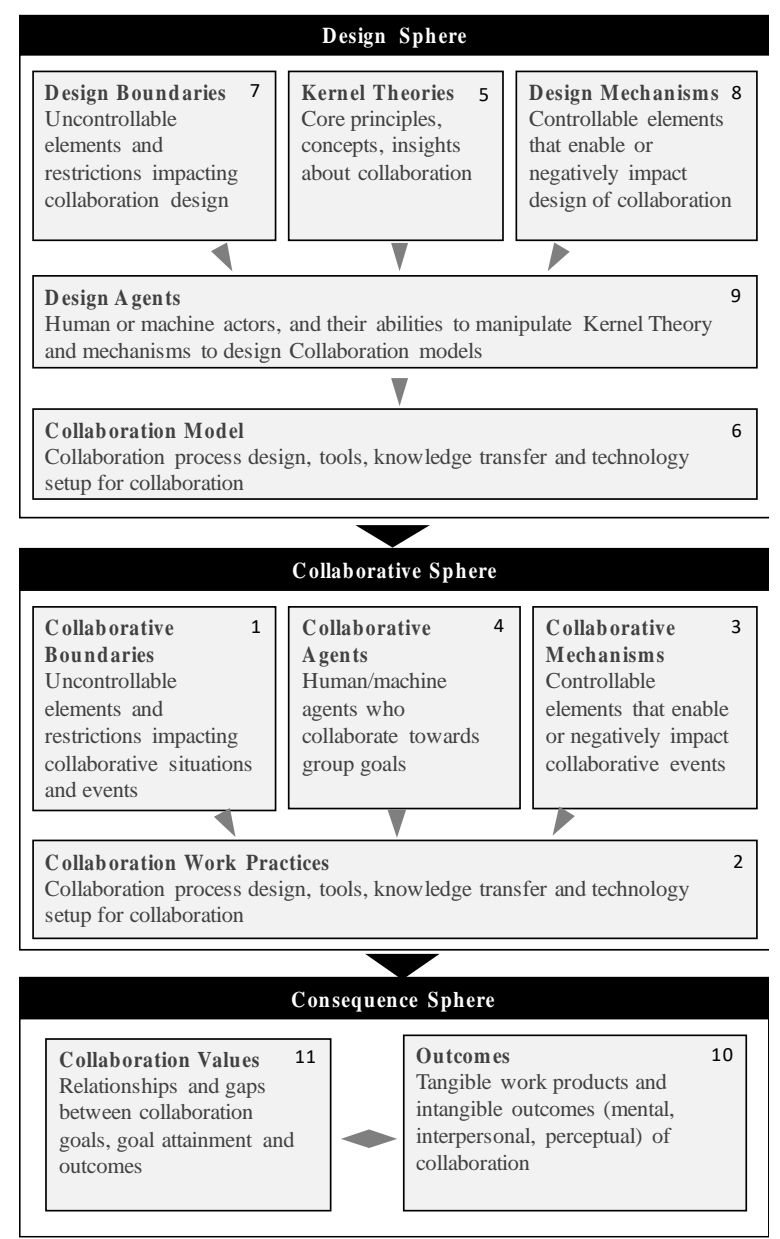

Figure 2: Reference model for the collaboration research agenda

Examples of boundaries include culture, availability of resources, and time. Contextual boundaries shape any kind of collaboration process and therefore impact the collaboration effort. Research questions on boundaries could include:

- How should organizational structures adopt routines, standards, and norms for smart technologies?

- How does acceptance of machine agents and their recommendations vary by demographics, by education, or by culture?

- What impacts do different smart technology enhanced collaboration environments (e.g., shop floor, self-driving cars) have on collaboration?

- What economic, political, social, cognitive, emotional, or physical considerations might impede 
or enhance value people derive by collaborating with machines?

Collaborative Work Practices (Theme 2) describes the research questions related to actual observable efforts, actions, interactions, and behavior undertaken by the actors during the collaborative process. The collaboration work practices relate to the actual behavior exhibited by the collaborating agents (participants, facilitators, collaboration supporters etc.) who engage in collaborative efforts. This behavior may or may not be helpful toward the expected collaborative consequences. Research questions regarding collaborative work practices include:

- To what extend can groups rely on the objectivity of intelligent technology agent's data input?

- To what extent might intelligent agents be susceptible to deliberately misleading information, lies, equivocations, alternative facts and fake news?

- How can we decrease counterproductive cognitive, affective, and behavioral emergent states with smart technology management?

- How does the introduction of smart technology in a collaborative work practice affect existing power and control relationships?

Collaborative Mechanisms (Theme 3) describe the research questions related to concepts that can be manipulated to improve or hinder the efficiency and effectiveness of the collaboration work practices. It may be useful to catalogue and understand these mechanisms within the scope of collaborative work practice design make better decisions about how to use the new technologies for optimum benefits. Examples of mechanisms include cultural awareness, communication style and leadership style, group size, collaboration space, technology usage, reward systems etc. Research questions on collaborative mechanisms include:

- How should human or machine agents mediate differences of cultural background (e.g. language, values, interpretations, goals, preferences).

- How should worker knowledge, skills, and abilities evolve to accommodate the new reality?

- To what extent will it be possible or useful for robots to develop social relationships with human teammates? Will a robot body capable of social interactions be better teammates than a disembodied, and possibly voiceless algorithm?

- How should intelligent agents manipulate large real time data flows into actionable insights during collaborative work practices?
Collaborative Agents (Theme 4) relate to research questions about the nature, availability, and capabilities of actors who participate in collaborative events. Agents include a variety of actors and roles in collaborative events, such as collaborators, facilitators, supporters, and observers, both human and machine. Agents represent the set of stakeholders who directly participate in the collaborative events. Research questions about the structure and composition of collaborative technologies also reside under this theme. Research questions on collaborative agents include:

- What are the prerequisite skills and personality traits needed for valuable participation in collaborative events?

- How can smart technology agents ensure data protection when acting upon personal and private data gathered from humans in the collaborative effort?

- How will human leaders deal with and accept highperforming, super productive, always compliant, and healthy digital agents compared to regular human agents?

- Would it be possible for digital agents to assist humans to transition from ideological thinking to critical thinking?

\subsection{Design Sphere (Meta-Theme B)}

The Design Sphere concerns the efforts and outcomes of defining, planning, and designing collaborative work practices for others to execute. The output of the Design Sphere acts as a guide and foundation for the efforts that take place in the collaborative sphere.

Kernel Theories (Theme 5) are models that predict and explain observed variations in the outcomes we seek to improve by designing collaboration processes. They inform and guide the principles and practices of a design domain. Collaboration Engineering, for example, is informed by theories of group productivity, creativity, satisfaction, willingness-to-change, learning, cognitive load, and other key phenomena by which the success or failure of collaboration are determined. Kernel theories create the foundation for assumptions and hypotheses regarding the causes, effects, and relationships between constructs, thus allowing collaboration engineers to predict counter-intuitive consequences for process-design choices. An example of a kernel theory is Goal Setting Theory [24]. There already exists a wide variety of Kernel Theories. Examples of additional research questions for expansion of the pool of important Kernel Theories include: 
- What new phenomena will emerge as humans begin to collaborate with $\mathrm{AI}$, that may require the development of new kernel theories? What old kernel theories may prove inadequate as known phenomena manifest in new, problematic ways?

- What changes may be required to CE development methodologies to address new concerns that emerge from smart technology/human partnerships? What constitutes the Collaboration Engineering Book of Knowledge?

- Is it possible to create a single, canonical ontology for collaboration? How has the ontology of collaboration developed over time? How might it need to evolve in the future?

Collaboration Model (Theme 6) describes the research questions related to the engineering of collaborative work practices in terms of processes, collaboration techniques, and behavior to elicit desired collaboration outcomes. A collaboration model is the core outcome of the design phase, which provides a template and roadmap to capture specific recommendations for a purposeful execution of the collaboration effort. The Six Layer Model [23] is an example of a collaboration model for which the collaboration designers recommend specific actions for each design layer to be used within the collaborative effort. Research questions with regard to the collaboration model include:

- How can collaboration mining and machine learning be used to derive design guidelines and best practices for future uses?

- How does smart technology affect each of the six layers of collaboration design?

- How will smart technology alter the techniques to generate, converge, organize, evaluate, and build commitment as well as the content of group deliverables from crowds?

- What is an appropriate documentation format (FPM, internal agenda) for collaborative process that incorporates smart technologies and new modes of collaboration?

- How should we model and design hierarchical interaction between and division of labor among smart technology and human agents?

Design Boundaries (Theme 7) describe the research questions related to the overarching environment under which the collaboration design constructs interact with each other. It is similar in nature to collaborative boundaries, but significantly different because design boundaries include factors that restrict the design efforts rather than the collaborative efforts. The boundaries cover all environmental concepts that cannot be manipulated or controlled within the timespan of the collaboration design. A boundary represents both a restriction and an opportunity for a collaboration engineer. Research questions on boundaries in the design sphere include:

- How do organizational policies, legal restrictions, and cultural norms, values, and behavioral expectations constrain the design of collaboration leveraging smart technologies? How do they advance and enhance the design of collaboration leveraging smart technology?

- What prioritizations, developments, funding, and knowledge sharing are required to increase the acceptance of smart technology in the design of collaboration? What as-yet unnoticed tacit assumptions may block or advance the diffusion of smart technology into collaboration roles?

Design Mechanisms (Theme 8) describe the research questions related to concepts that can be manipulated and have the potential to affect the efficiency and effectiveness of the collaboration model design. Mechanisms are controllable factors that can be invoked to maximize the quality and value of the collaboration model. These are similar in nature to collaborative mechanisms, but differ as they relate exclusively to the design of standardized collaboration models and not the execution of collaborative events. While design boundaries and design mechanisms have direct implications for the design of collaboration, design mechanisms only have an indirect influence through the collaboration model on the collaboration work practices. Research questions on mechanisms in the design sphere include:

- What usability design concerns result from smart technology partners and crowds?

- How can innovative technologies be used to assist and control collaboration design work?

- Which smart technology-enabled process restrictions are appropriate to restrict designers of collaboration to focus on the appropriate collaboration model design task?

Design Agents (Theme 9) relate to research questions about the nature, availability and capabilities of collaboration designer and engineering experts who design standardized collaboration models. Agents include collaboration engineering professionals, both human and machine, and research questions about their roles and responsibilities in the collaboration design. Design agents represent the set of stakeholders who directly participate in the design of collaboration models. Research questions on design agents include: - What are the prerequisite skills, capabilities, experiences, and personality traits needed to be considered a professional collaboration engineer? 
- What collaboration design tasks can smart technology perform and which should be performed by humans?

- What is are appropriate architecture for machinebased collaboration design agents and providers?

\subsection{Consequence Sphere (Meta-theme C)}

A final meta-theme emerging relates to the consequences of collaboration. Consequences describe research questions related to outcomes in the form of direct output and deliverables (e.g. work products and targeted intangible outcomes) and values of the outcomes. Consequences research in essence deals with the research of results and their drivers and restrictors. Consequences are the result of the sequence of steps taken to design and execute the collaboration. Consequence research documents both positive and negative consequences as well as expected and unexpected consequences.

Outcomes (Theme 10) relate to research questions related to the direct tangible work products and concrete deliverables. It also includes the direct intangible outcomes in the form of mental, interpersonal, perceptional, or even spiritual consequences of the collaborative events. Research questions about outcomes include, for instance:

- How could intangible outcomes be measured beyond the perceived satisfaction and perceived goal attainment?

- What measure of other cognitive or sub-conscious effects could be useful to quantify the benefits or costs of smart technology/human collaboration?

Collaboration Values (Theme 11) concern research questions related to the value of collaboration in the form of benefits of the outcomes to different stakeholders. Values can be defined in terms of financial and non-financial values for stakeholders. Non-financial values include, for instance, better personal relationships, sustainability, strategy alignment, learning, and growth. Stakeholder value perspectives include individuals (humans, machines, designers and collaborators), groups, organizations, networks, and societies. Value research includes alignment between stakeholder goals, design objectives, outcomes, and goal attainment. Research questions about collaboration values include:

- What is the financial value of different types of tangible work products?

- How do different stakeholders' goals and value perspectives differ? And which stakeholder value expectations should be prioritized?

To summarize, we identified a conceptual relationship between the themes based on an analysis of the nature and inter-relationships between the emerged and identified themes. This discovery led to the development of a conceptual reference model, which can show how the themes relate to each other (see Figure 2). The model consists of a design -based sphere which includes design boundaries, kernel theory, design mechanisms, design agents, and collaboration models themes. It furthermore consists of a collaborative sphere, comprising collaborative boundaries, agents, mechanisms, and work practice themes. Finally, it consists of a consequence sphere, which includes collaboration outcomes and values.

The arrows in the model suggest associations among the elements and not yet any cause and effect relationships. Using the reference model, researchers can instantiate themes with relevant theoretical research concepts and relate these concepts to causal relationships. Researchers could then investigate the impact of contexts, mechanisms, use of kernel theory, collaboration models, collaboration effort, and collaboration agents on outcomes. Investigations could also study the relationship between desired and actual goal attainment identification/explanation for any gaps between the two. Hence, investigating causal relationships might span different spheres but could also connect concepts within a single sphere with different themes. The model can also help partners in practice to help structuring their change management activities as their business as well as their human capital needs to adapt to the new ways of working.

Naturally, we cannot yet claim that the reference model is fully comprehensive and covers all potential research questions. The sample of researchers contributing to the results presented in this paper is not equal to all the collaboration researchers and other stakeholders in collaboration research that might have additional relevant research questions. Stakeholders in addition to collaboration researchers were not directly involved in analyzing technology consequences or suggesting collaboration scenarios or relevant research questions. The reference model does, however, present a new comprehensive overview of the major areas of research, which can unveil and contribute new relevant knowledge and insights about collaboration for the benefit of scientific progress.

\section{Conclusion}

The goal of this paper was to develop a research agenda outlining open questions for collaboration researchers. We outlined a collaboration research agenda consisting of 3 meta- and 12 sub-themes illustrated in a reference model. This research agenda is therefore our major contribution to directly answer 
the overall research question for how collaboration researchers shall prepare for machines as teammates. Our research is intended to be a foundational reference model to guide researchers' efforts in collaboration research being it analytical/conceptual and empirical research approaches. We also contribute a set of future collaboration scenarios, a list of collaboration research challenges, and selected research questions to help kick-off future collaboration research.

\section{Acknowledgements}

We thank our research colleagues Daniel Mittelman and Xusen Cheng for their critical feedback. The research was partially funded by the Austrian Science Foundation (FWF): P 29765

\section{References}

[1] D. Norman, "Design , Business Models , and Human-Technology Teamwork," Res. Manag., vol. 60, no. 1, pp. 26-30, 2017.

[2] M. B. Rosson and J. M. Carroll, Usability engineering: scenario-based development of human-computer interaction. Morgan Kaufmann, 2001.

[3] M. B. Rosson and J. M. Carroll, "Scenario-based design," Human-Computer Interact. Handbook, 2nd Ed., 2007.

[4] A. Dennis and B. Wixom, "Investigating the moderators of the group support systems use with meta-analysis," J. Manag. Inf. Syst., vol. 18, no. 3, pp. 235-257, 2002.

[5] J. Fjermestad and S. Hiltz, "An assessment of group support systems experimental research: methodology and results," J. Manag. Inf. Syst., vol. 15 , no. 3 , pp. 7-149, 1998.

[6] J. F. Nunamaker, A. R. Dennis, J. S. Valacich, D. R. Vogel, and J. F. George, "Electronic Meeting Systems to Support Group Work," Commun. ACM, vol. 34, no. 7, pp. 40-61, 1991.

[7] R. O. Briggs and G.-J. de Vreede, Facilitation of Technology Supported Collaboration. The Center for Collaboration Science, 2001.

[8] A. Dennis and M. Garfield, "The adoption and use of GSS in project teams: Toward more participative processes and outcomes," Mis $Q$., vol. 27, no. 2, pp. 289-323, 2003.

[9] V. Huff Eckert, C. Curran, and S. C. Bhardwaj, "Tech breakthroughs megatrend: how to prepare for its impact," 2016.

[10] Artificial Intelligence, "Merr," MerriamWebster.com, 2011. [Online]. Available: https://www.merriam-

webster.com/dictionary/artificial intelligence. [Accessed: 08-Jun-2017].
[11] G. Rometty, "Digital Today, Cognitive Tomorrow.," MIT Sloan Manag. Rev., vol. 58, no. 1, pp. 168-171, 2016.

[12] W. Frick, "When Your Boss Wears Metal Pants," Harv. Bus. Rev., no. June, pp. 84-90, 2015.

[13] R. Hoffman, "Using Artificial Intelligence to Set Information Free.," MIT Sloan Manag. Rev., vol. 58, no. 1, pp. 1-5, 2016.

[14] P. J. H. Schoemaker and Philip E. Tetlock, "Building a More Intelligent Enterprise," MIT Sloan Manag. Rev., pp. 1-15, 2017.

[15] M. Dowd, "Elon Musk's Billion-Dollar Crusade to Stop the A.I. Apocalypse," Vanity Fair, pp. 119, 2017.

[16] K. Kelly, "Better Than Human: Why Robots Will - And Must - Take Our Jobs," Wired, pp. 1-16, 2012.

[17] E. Brynjolfsson and A. P. McAfee, The second machine age. Norton Paperback, 2014.

[18] D. L. Dean, J. M. Hender, T. L. Rodgers, and E. L. Santanen, "Identifying quality, novel, and creative ideas: Constructs and scales for idea evaluation," J. Assoc. Inf. Syst., vol. 7, no. 10, pp. 646-698, 2006.

[19] R. O. Briggs, G.-J. de Vreede, and J. A. Nunamaker, "Collaboration Engineering with ThinkLets to Pursue Sustained Success with Group Support Systems," J. Manag. Inf. Syst., vol. 19, no. 4, pp. 31-64, 2003.

[20] G. J. de Vreede, R. O. Briggs, and A. P. Massey, "Collaboration engineering: foundations and opportunities: editorial to the special issue on the journal of the association of information systems," J. Assoc. Inf. Syst., vol. 10, no. 3, p. 7, 2009.

[21] R. Briggs and G. J. de Vreede, ThinkLets: Building Blocks for Concerted Collaboration. 2009.

[22] G. L. Kolfschoten and G.-J. de Vreede, "A Design Approach for Collaboration Processes: A Multimethod Design Science Study in Collaboration Engineering," J. Manag. Inf. Syst., vol. 26, no. 1, pp. 225-256, Jul. 2009.

[23] R. O. Briggs, G. L. Kolfschoten, G. de Vreede, S. Lukosch, and D. L. Dean, "A six-layer model of collaboration.," in Collaborative Systems: Concept, Value, and Use, London: Taylor \& Francis Group, 2015, pp. 211-227.

[24] E. Locke and G. Latham, "Goal-setting theory," in Organizational Behavior 1: Essential Theories of Motivation and Leadership, J. B. Miner, Ed. Hillsdale, NJ, US: Lawrence Erlbaum Associates, 1994, pp. 159-183. 